\title{
Raman spectroscopy of GaP/GaNP core/shell nanowires
}

A. Dobrovolsky, S. Sukrittanon, Y. J. Kuang, C. W. Tu, Weimin Chen and Irina Buyanova

\section{Linköping University Post Print}

\section{Tweet}

N.B.: When citing this work, cite the original article.

Original Publication:

A. Dobrovolsky, S. Sukrittanon, Y. J. Kuang, C. W. Tu, Weimin Chen and Irina Buyanova, Raman spectroscopy of GaP/GaNP core/shell nanowires, 2014, Applied Physics Letters, (105), 19, 193102.

http://dx.doi.org/10.1063/1.4901446

Copyright: American Institute of Physics (AIP) http://www.aip.org/

Postprint available at: Linköping University Electronic Press

http://urn.kb.se/resolve?urn=urn:nbn:se:liu:diva-113015 




\section{Raman spectroscopy of GaP/GaNP core/shell nanowires}

A. Dobrovolsky, S. Sukrittanon, Y. J. Kuang, C. W. Tu, W. M. Chen, and I. A. Buyanova

Citation: Applied Physics Letters 105, 193102 (2014); doi: 10.1063/1.4901446

View online: http://dx.doi.org/10.1063/1.4901446

View Table of Contents: http://scitation.aip.org/content/aip/journal/apl/105/19?ver=pdfcov

Published by the AIP Publishing

\section{Articles you may be interested in}

Growth and characterization of dilute nitride GaNxP1-x nanowires and GaNxP1-x/GaNyP1-y core/shell nanowires on $\mathrm{Si}(111)$ by gas source molecular beam epitaxy

Appl. Phys. Lett. 105, 072107 (2014); 10.1063/1.4893745

Micro-Raman investigations of InN-GaN core-shell nanowires on $\mathrm{Si}$ (111) substrate

AIP Advances 3, 062114 (2013); 10.1063/1.4811365

Growth and characterization of GaP/GaNP core/shell nanowires

J. Vac. Sci. Technol. B 31, 03C110 (2013); 10.1116/1.4793476

Mechanism for radiative recombination and defect properties of GaP/GaNP core/shell nanowires Appl. Phys. Lett. 101, 163106 (2012); 10.1063/1.4760273

Growth and photoluminescence of self-catalyzed GaP/GaNP core/shell nanowires on $\mathrm{Si}(111)$ by gas source molecular beam epitaxy

Appl. Phys. Lett. 100, 053108 (2012); 10.1063/1.3681172 


\title{
Raman spectroscopy of GaP/GaNP core/shell nanowires
}

\author{
A. Dobrovolsky, ${ }^{1}$ S. Sukrittanon, ${ }^{2}$ Y. J. Kuang, ${ }^{3}$ C. W. Tu, ${ }^{4}$ W. M. Chen, ${ }^{1}$ \\ and I. A. Buyanova ${ }^{1, a)}$ \\ ${ }^{1}$ Department of Physics, Chemistry and Biology, Linköping University, S-581 83 Linköping, Sweden \\ ${ }^{2}$ Graduate Program of Materials Science and Engineering, La Jolla, California 92093, USA \\ ${ }^{3}$ Department of Physics, University of California, La Jolla, California 92093, USA \\ ${ }^{4}$ Department of Electrical and Computer Engineering, University of California, La Jolla, \\ California 92093, USA
}

(Received 26 September 2014; accepted 30 October 2014; published online 10 November 2014)

\begin{abstract}
Raman spectroscopy is employed to characterize structural and phonon properties of GaP/GaNP core/shell nanowires (NWs) grown by molecular beam epitaxy on Si substrates. According to polarization-dependent measurements performed on single NWs, the dominant Raman modes associated with zone-center optical phonons obey selection rules in a zinc-blende lattice, confirming high crystalline quality of the NWs. Two additional modes at 360 and $397 \mathrm{~cm}^{-1}$ that are specific to the NW architecture are also detected in resonant Raman spectra and are attributed to defectactivated scattering involving zone-edge transverse optical phonons and surface optical phonons, respectively. It is concluded that the formation of the involved defect states are mainly promoted during the NW growth with a high V/III ratio. (C) 2014 AIP Publishing LLC.
\end{abstract}

[http://dx.doi.org/10.1063/1.4901446]

III-V semiconductor nanowires (NWs) are currently attracting considerable attention as one of the most promising architectures for a variety of nanoscale devices, including solar cells, light-emitting diodes (LEDs), and photo-detectors. ${ }^{1-4}$ Recent advances in growth techniques have made it possible to vary chemical compositions either axially along the NW's length or radially, i.e., by growing surrounding shells of different materials resulting in core/shell NW structures. ${ }^{5-7}$ The latter approach is very promising for fabrication of photovoltaic devices, since it can improve the efficiency of optical absorption and charge collection in vertically aligned NW arrays. ${ }^{8}$ A promising material system attractive for applications in solar cells and light-emitting diodes is $\mathrm{GaN}_{\mathrm{x}} \mathrm{P}_{1-\mathrm{x}} /$ $\mathrm{GaN}_{\mathrm{y}} \mathrm{P}_{1-\mathrm{y}}$ core/shell NWs. ${ }^{9-12}$ Incorporation of several percent of nitrogen in gallium phosphide reduces its lattice constant, thereby minimizing lattice mismatch with $\mathrm{Si}^{13}{ }^{13}$ Also, a strong interaction between the $\mathrm{N}$-related localized states and the extended states of GaP modifies the band structure of the forming alloy leading to an increased oscillator strength of the band-to-band optical transitions ${ }^{14-16}$ and a splitting of the conduction band states into two subbands. ${ }^{15,17}$ The former is advantageous for utilizing this material in amber light emitting diodes, whereas the latter makes GaNP an attractive material for applications in innovative intermediate-band solar cells with an anticipated high efficiency. ${ }^{18}$

Growth of NWs is often accompanied by polytypism and formation of various point and planar defects, such as rotational twinning planes and stacking faults, which can affect functionality of electronic and photonic devices based on NWs. ${ }^{19,20}$ An effective technique that allows non-destructive characterization of electronic and structural properties of nanoscale materials is micro-Raman spectroscopy. This technique has been applied to several III-V NW systems ${ }^{19-27}$ and

\footnotetext{
${ }^{\text {a) }}$ Author to whom correspondence should be addressed. Electronic mail: iribu@ifm.liu.se
}

provided important information regarding crystalline structure, such as presence of staking disorder and formation of alternating zinc-blende (ZB)/wurtzite (WZ) (ZB/WZ) polytypes, strain as well as diameter modulations. The purpose of this work is by using Raman spectroscopy to characterize structural and phonon properties of recently developed coaxial GaP/GaNP NWs that are currently unknown.

The investigated $\mathrm{GaP} / \mathrm{GaNP}$ core/shell NWs were grown by gas-source molecular beam epitaxy (GS MBE) on (111)oriented Si substrates using as a catalyst Ga droplets formed on the substrate surface. ${ }^{9}$ As a reference, GaP NWs were also studied. The latter were grown under the vapor-liquid-solid (VLS) mechanism at substrate temperatures $\mathrm{T}_{\text {sub }}$ of $580-615^{\circ} \mathrm{C}$ with a V/III flux ratio being varied from 1.5 to 6.9 for different samples. The same growth procedure was used for the GaP core layer in the coaxial NW structures, where the V/III ratio was kept at around 1.5. The GaNP shells were fabricated via the step-mediated growth performed at $\mathrm{T}_{\text {sub }}$ of $520^{\circ} \mathrm{C}$ with a nitrogen plasma ignited. During the shell growth, the $\mathrm{V} / \mathrm{III}$ ratio was increased to 2.5 . The nitrogen composition in the shell was estimated to be around $0.9 \%$ from room-temperature photoluminescence data using the band anti-crossing model. The detailed growth conditions can be found in Ref. 9. The NWs are rather uniform in sizes and have an axial length of about $2-2.5 \mu \mathrm{m}$, average diameters ranging from 80 to $110 \mathrm{~nm}$ for the GaP NWs (depending on the $\mathrm{V} / \mathrm{III}$ ratio) and a typical diameter of approximately $220 \mathrm{~nm}$ for the GaP/GaNP core/shell NWs. They are predominantly oriented along the [111] direction. According to highresolution transmission electron microscopy (HRTEM), both $\mathrm{GaP}$ and $\mathrm{GaP} / \mathrm{GaNP}$ core/shell NWs predominantly have the ZB structure with a rather high density of random twins/ stacking faults perpendicular to the growth axis. ${ }^{12}$ The contribution of the hexagonal WZ phase in a single NW was typically below $5 \%$ of the whole volume. For a comparison, a 750 -nm-thick $\mathrm{GaN}_{0.009} \mathrm{P}_{0.991}$ epilayer grown by gas-source 
MBE on a (001)-oriented GaP substrate was also investigated. Raman measurements were performed at room temperature using a Horiba Jobin Yvon micro-Raman spectrometer under excitation by the $325 \mathrm{~nm}$ line of a He-Cd laser or the $659 \mathrm{~nm}$ line of a solid-state laser. The measurements were conducted in the back scattering geometry on the NW arrays and individual NWs that were mechanically transferred onto another Si substrate.

Fig. 1(a) presents Raman spectra from the GaP and GaP/ GaNP NW arrays measured under the $325 \mathrm{~nm}$ excitation. For all investigated structures, the spectra contain the transverse (TO) and longitudinal (LO) phonon modes due to the zonecenter $(\Gamma)$ optical phonons in $\mathrm{ZB} \mathrm{GaP} .{ }^{28}$ The formation of the GaNP shell leads to the appearance of an additional Raman peak at around $500 \mathrm{~cm}^{-1}$ (labelled as $\mathrm{LO}_{2}$ in Fig. 1(a)). This mode was previously detected in GaNP epilayers and attributed to Ga-N bond vibrations in the GaNP alloy that shows a bimodal behavior. ${ }^{28}$ Its observation in the studied core/shell structures provides an independent proof for the formation of the GaNP shell. In the core/shell structures, a change of excitation wavelength $\left(\lambda_{\text {exc }}\right)$ to $659 \mathrm{~nm}$ leads to enhancement of several additional Raman modes at $\sim 360$, 387 , and $397 \mathrm{~cm}^{-1}$ (Fig. 1(b)). The mode at $387 \mathrm{~cm}^{-1}$ (labeled as X) is also observed in the Raman spectra of the GaNP epilayer (the dotted curve in Fig. 1(b)), in accordance with previous findings. ${ }^{28,29}$ Its energy is very close to the LO phonon energy at the X-point of the Brillouin zone of GaP. ${ }^{30}$ The activation of this forbidden mode can be explained by relaxation of the momentum conservation rules as a result of breaking of translational symmetry by incorporation of

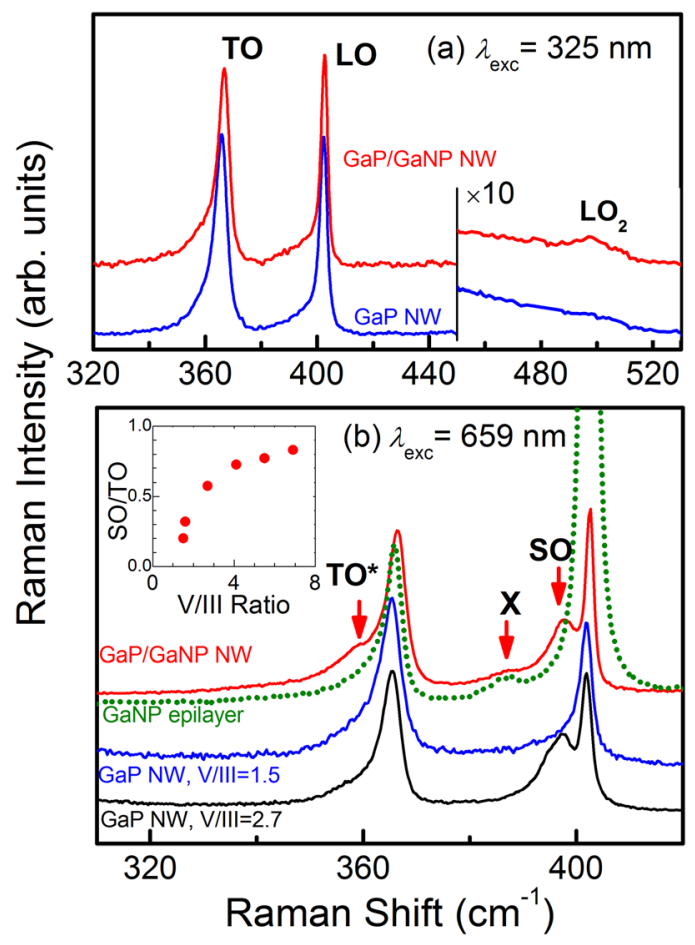

FIG. 1. Raman spectra measured from the GaP NW and the GaP/GaNP NW (the solid curves) arrays, as well as from the reference GaNP epilayer (the green dotted curve) using $\lambda_{\text {exc }}$ of $325 \mathrm{~nm}$ (a) and $659 \mathrm{~nm}$ (b), respectively. The spectra are normalized to the intensity of the TO mode and are shifted vertically for clarity. The inset in (b) shows intensity of the SO mode, normalized to that of the TO mode, as a function of the V/III ratio utilized during the NW growth. nitrogen. As to the $360 \mathrm{~cm}^{-1}$ (labeled as TO*) and $397 \mathrm{~cm}^{-1}$ (labeled as SO) Raman peaks, they are not detected from the GaNP epilayers, which implies that these modes become active only in the NW structures. From Fig. 1(b), it is obvious that both 360 and $397 \mathrm{~cm}^{-1}$ modes in the GaP/GaNP core/shell structures stem from the GaNP shell, not from the $\mathrm{GaP}$ core, as they could not be detected from the reference GaP NWs grown with the same V/III ratio (1.5) as the GaP core. This activation could be either directly related to the presence of nitrogen or caused by variation of growth conditions, e.g., an increased V/III ratio of 2.5, utilized during the growth of the GaNP shell. To clarify this issue, we have studied Raman properties of GaP NWs grown with a comparable V/III ratio of 2.7 but without $\mathrm{N}$ incorporation-see Fig. 1(b). Obviously, this change in the growth conditions also results in the appearance of two Raman peaks located at the low energy side of the TO and LO modes. Their intensity increases with increasing V/III ratio, as shown in the inset in Fig. 1(b) taking as an example the $397 \mathrm{~cm}^{-1}$ mode.

The reasons for the appearance of the additional Raman modes in the NWs can be twofold. First of all, increasing surface-to-volume ratio in the NW structures allows crystallization of III-V materials in the WZ phase which has a lower surface energy than $\mathrm{ZB} .{ }^{31}$ With respect to $\mathrm{ZB}$, the $\mathrm{WZ}$ crystal structure should lead to activation of two additional optical phonon modes $\left(\mathrm{E}_{2}{ }^{\mathrm{h}}\right.$ and $\left.\mathrm{B}_{1}{ }^{\mathrm{h}}\right)$ at the $\Gamma$ point of the Brillouin zone that stem from the L-point optical phonons in $\mathrm{ZB}{ }^{23,25}$ This is a consequence of the back folding of the phonon dispersion due to doubling of the unit cell length in the [111] direction. Since the Raman active phonons in $\mathrm{ZB}$ have $\mathrm{A}_{1}$, $\mathrm{E}_{1}$, and $\mathrm{E}_{2}$ symmetry, only the $\mathrm{E}_{2}{ }^{\mathrm{h}}$ mode is Raman active and should be located at the low energy side of the TO mode, based on phonon dispersion. The appearance of a Raman band at the low-energy side of the TO mode was previously observed in III-V NWs with WZ inclusions. ${ }^{23,32,33}$ The energy position of the $\mathrm{TO}^{*}$ peak in our samples is indeed very close to the energy of the $\mathrm{TO}(\mathrm{L})$ phonons in $\mathrm{GaP},{ }^{34}$ which makes this explanation plausible. Alternatively, additional modes could be activated due to breaking of translational symmetry caused by formation of either planar (e.g., twinning planes and mixed WZ/ZB segments) or point defects that relaxes Raman selection rules. In this case, energies of the activated modes will be determined by the phonon density of states, which has a maximum at around $356 \mathrm{~cm}^{-1}$ in bulk GaP, ${ }^{34}$ i.e., again in the vicinity of the TO* peak.

The aforementioned two alternatives could be distinguished by analyzing polarization properties of the Raman modes, which are expected to obey selection rules only for unperturbed $\mathrm{WZ}$ and $\mathrm{ZB}$ lattices, i.e., within the first scenario. Given that all Raman modes are allowed in the backscattering geometry from the NW arrays, polarizationdependent Raman measurements were performed on a single NW. Since the investigated NWs exhibit a hexagonal crosssection with the NW axis along the [111] direction, the top flat facet of the $\{1 \overline{1} 0\}$ family will be perpendicular to the incident light. The coordinate system is then chosen according to the NW geometry with the $x$ axis along the [1 $\overline{1} 0]$ direction of the incident light, while $y([11 \overline{2}])$ and $z$ ([111]) are the in-plane axes that are perpendicular and parallel to the NW axis, respectively-see the inset in Fig. 2(a). The 
results from the polarization-dependent measurements are summarized in Figs. 2(a) and 2(b), which show Raman spectra obtained under four main polarization configurations and azimuthal dependences of the most pronounced Raman modes, respectively. The dominant TO mode is found to have the highest intensity in the $x(z, z) \bar{x}$ and $x(y, y) \bar{x}$ polarizations, consistent with the Raman selection rules for $\mathrm{ZB} .{ }^{23}$ The somewhat weaker intensity of this mode in the $x(y, y) \bar{x}$ configuration could be attributed to the so-called antenna effect, ${ }^{35,36}$ which may cause a suppression in the electric field of the electromagnetic waves of the light inside the nanowire when the field direction is perpendicular to its axis. (We note, however, that the antenna effect is not expected to be very pronounced in the studied wires due to the relatively large NW diameter $\mathrm{d} \sim \lambda_{\text {exc }} / 4$.) The observed TO polarization behaviour implies that the studied NWs have a good crystalline quality so that the Raman selection rules are generally obeyed. On the other hand, the LO mode is forbidden in the backscattering geometry from $\{1 \overline{1} 0\}$ surfaces. This mode is clearly visible in our structures, though weak, and is practically isotropic in polarization-see Fig. 2(b). A probable reason for the appearance of this mode is micro faceting of the NW surface in regions with a high density of twin planes, which results in its termination by $\{111\}$ facets $^{37}$ for which the LO mode is allowed. This formation of micro facets in the investigated NWs was confirmed from the performed TEM measurements. ${ }^{12}$ As to the TO* mode, it has the highest intensity in the $x(z, z) \bar{x}$ configuration, i.e., it does not follow the selection rules for WZ. ${ }^{23}$ Moreover, a

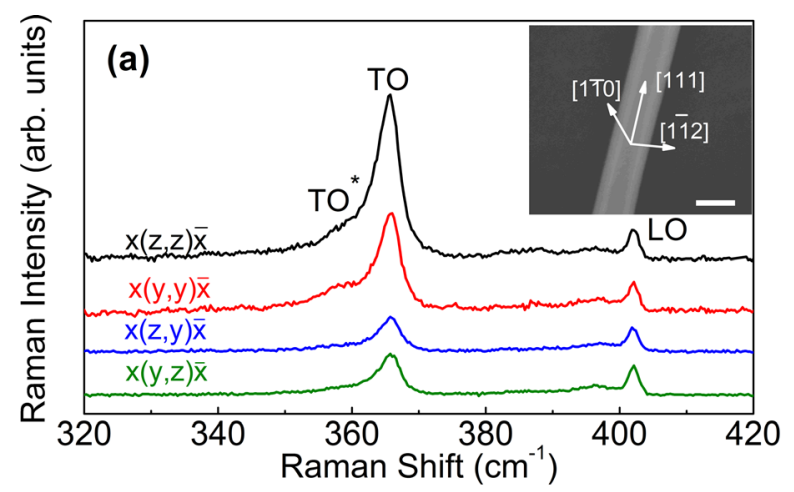

(b)

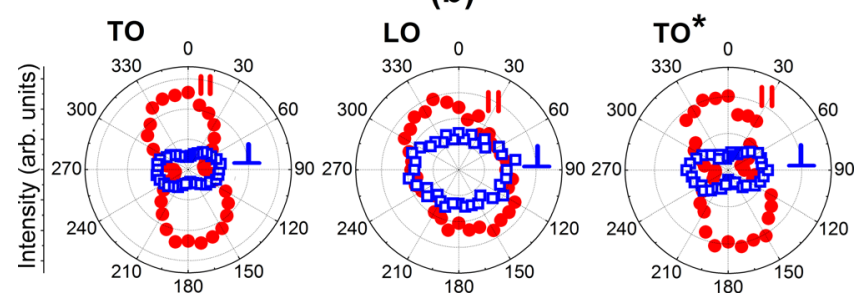

FIG. 2. (a) Raman spectra of a single GaP/GaNP NW measured in the specified polarization configurations. The spectra are vertically offset, for clarity. The inset shows an SEM image of the GaP/GaNP NW transferred onto a Si substrate, with a scale bar of $200 \mathrm{~nm}$. The set of reference axes $\mathrm{x}, \mathrm{y}$, and $\mathrm{z}$ is indicated in the image. (b) Azimuthal dependences of the Raman modes. Intensities of the $360 \mathrm{~cm}^{-1}$ mode were determined by de-convoluting the measured spectra assuming a Lorentzian line shape for each individual Raman peak. The red filled circles and the blue open squares represent intensities of the Raman components that are polarized parallel and perpendicular to the NW axis, respectively. comparison of the Raman spectra presented in Figs. 1(a) and 1(b) shows that the mode is enhanced under the $659 \mathrm{~nm}$ excitation, i.e., likely exhibiting a resonant behavior with respect to the excitation wavelength. The resonant enhancement of the Raman scattering efficiency occurs when the scattering process involves a real state, e.g., a defect-related state. We therefore suggest that this mode in the investigated NWs does not stem from the WZ regions, but it could represent defect-mediated scattering involving zone-edge TO phonons. We also note that the spectral position of this mode shifts to lower energies by $1-3 \mathrm{~cm}^{-1}$ with increasing the V/III ratio, which may indicate that it is influenced by defect density.

Let us now discuss the origin of the Raman peak that appears at $397 \mathrm{~cm}^{-1}$, seen in Fig. 1(b) on the low energy side of the LO mode under $\lambda_{\mathrm{exc}}=659 \mathrm{~nm}$. The most pronounced feature of this mode is its sensitivity to the dielectric constant of an external medium $\varepsilon_{m}$ that is in contact with the NWs. Fig. 3 presents the Raman spectra collected from the GaP/ GaNP (a) and GaP (b) NW arrays in air $\left(\varepsilon_{m}=1\right)$ and submerged in dichloromethane $\left(\varepsilon_{m}=2\right)$. Apparently, the spectral position of the $397 \mathrm{~cm}^{-1}$ mode (labelled as SO in Fig. 3) downshifts with increasing the dielectric constant of the surrounding media, whereas the positions of the other modes remain constant. This behaviour is typical for the so-called surface optical (SO) phonons ${ }^{21,38-40}$ that can be activated by breakdown of translational symmetry at surfaces. The wavevector of the corresponding perturbing potential could be

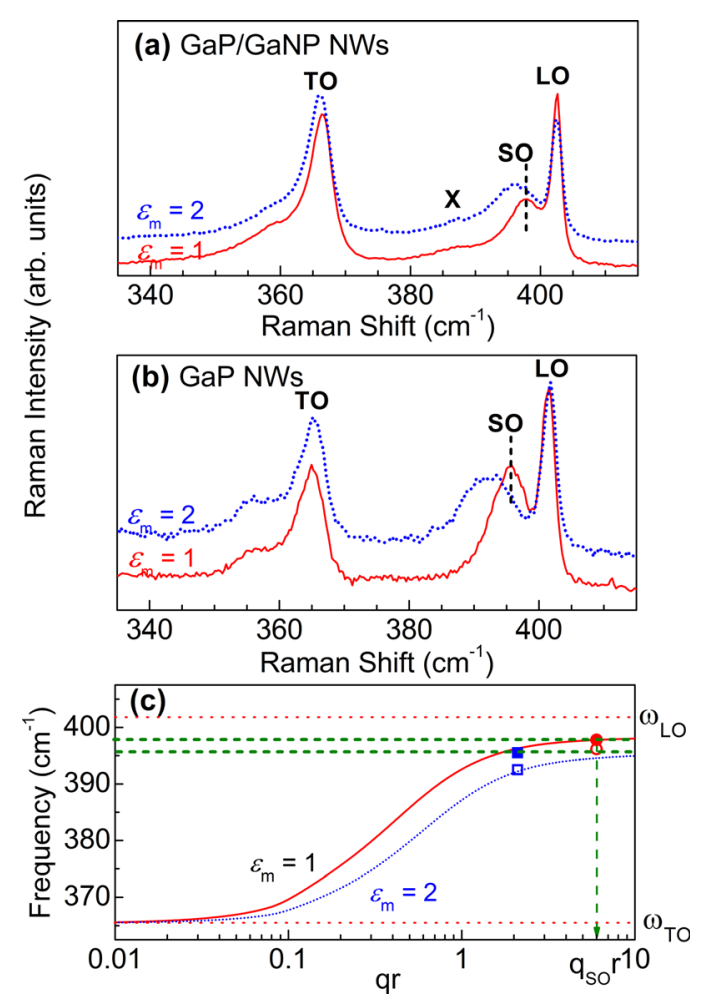

FIG. 3. Raman spectra of the GaP/GaNP NWs (a) and the GaP NWs grown with a V/III ratio of 5.5 (b) measured in two media with different dielectric constants $\varepsilon_{m}$ : air (the red solid curve) and dichloromethane (the blue dotted curve). (c) The calculated dispersion curves of the SO mode for the GaP/ GaNP and the GaP NWs in two different media. The symbols indicate the experimental values of the SO phonon frequencies detected from the GaP (the squares) and the GaP/GaNP NWs (the circles). The dashed green lines and arrow are guides to the eye. 
determined based on an analysis of SO dispersion. Following the formalism discussed in Ref. 38, the dispersion relation $\omega_{S O}(q)$ in NWs with a hexagonal cross-section can be approximated by that of an infinitely long cylindrical wire. For the phonons with wave vectors $q \gg \omega / c$, the SO dispersion is given by

$$
\omega_{S O}^{2}=\omega_{T O}^{2}+\frac{\tilde{\omega}_{p}^{2}}{\varepsilon_{\infty}+\varepsilon_{m} f(x)} .
$$

Here, $x=q r, \omega_{T O}$ is the TO phonon frequency, $\tilde{\omega}_{p}$ is the screened ion plasma frequency given by $\omega_{L O}^{2}=\omega_{T O}^{2}+\frac{\tilde{\omega}_{p}^{2}}{\varepsilon_{\infty}}, \varepsilon_{\infty}$ is the high-frequency dielectric constant of the material, $r$ is the NW radius, $f(x)=\left(I_{0}(x) K_{1}(x)\right) /\left(I_{1}(x) K_{0}(x)\right)$ with $I_{j}(x)$ and $K_{j}(x)$ being the Bessel functions. In Fig. 3(c), we plot the $\omega_{S O}(q r)$ dispersion for $\varepsilon_{m}=1$ (the red solid curve) and $\varepsilon_{m}=2$ (the blue dotted curve) taking $\varepsilon_{\infty}=9.075, \tilde{\omega}_{p}=502.28 \mathrm{~cm}^{-1}$, $\omega_{L O}=401.8 \mathrm{~cm}^{-1}$, and $\omega_{T O}=365.5 \mathrm{~cm}^{-1}$. The SO frequencies experimentally obtained in the GaP/GaNP NWs (the circles) and GaP NWs (the squares) in two dielectric media yield $q_{S O} r=6.1$ or $q_{S O}=0.055 \mathrm{~nm}^{-1}$ (with $r \sim 110 \mathrm{~nm}$ ) and $q_{S O} r=2.1$ or $q_{S O}=0.053 \mathrm{~nm}^{-1}$ (with $r \sim 40 \mathrm{~nm}$ ), respectively. The wavelength $\lambda_{S O}=2 \pi / q_{S O}$ corresponding to the perturbation of the surface potential can then be estimated as being $\sim 115 \mathrm{~nm}$. Several mechanisms were proposed ${ }^{21,39,40}$ to explain the SO activation in NWs, including surface roughness, saw tooth faceting on the NW sidewall, or a diameter modulation along the NW length on the scale of $\lambda_{\mathrm{SO}}$. None of these mechanisms, however, seem to be relevant to the studied structures. Indeed, according to our previous SEM and TEM studies, ${ }^{12}$ the NWs are uniform in diameter and show faceting on the scale of $5 \mathrm{~nm}$, which is by far smaller than the experimentally determined $\lambda_{S O}$. Instead, the momentum of the activated mode could correspond to the momentum transferred to the NW upon back scattering of the excitation light, i.e., $q=\frac{4 \pi \sqrt{\varepsilon}}{\lambda_{\text {exc }}}=0.060 \mathrm{~nm}^{-1}$ which is very close to the determined value of $q_{S O}$. Taking into account that the intensity of the SO mode is significantly enhanced under the $659 \mathrm{~nm}$ excitation, we suggest that its activation is again a result of resonant Raman scattering via defect states, possibly from point or structural defects located in the vicinity to the NW surfaces. The formation of these defects is not related to the presence of nitrogen, however, but it is facilitated when the NW growth is performed with high V/III ratios, based on the observed enhancement of the SO mode under such growth conditions (see the inset in Fig. 1(b)).

In conclusion, we have carried out detailed Raman measurements on the GaP NWs and GaP/GaNP core/shell NWs grown by molecular beam epitaxy on $\mathrm{Si}$ (111) substrates. The Raman scattering peaks related to the GaP-like LO and TO phonon modes are found to dominate the $320-420 \mathrm{~cm}^{-1}$ spectral range. The growth of the GaNP shell leads to the emergence of the $\mathrm{LO}_{2}$ mode at around $500 \mathrm{~cm}^{-1}$ due to the Ga-N bond vibrations in $\mathrm{GaP}$, which provides the evidence for $\mathrm{N}$ incorporation in the GaNP shell. The polarization-dependent Raman measurements performed on single NWs indicate that the TO Raman modes obey the selection rules in $\mathrm{ZB}$, confirming high crystalline quality of the NWs. Two additional bands at 360 and $397 \mathrm{~cm}^{-1}$ were also detected in the resonant Raman spectra, which could be attributed to the scattering involving the zone-edge TO phonons and a surface mode, respectively. The characteristic wavelength of the perturbation of the surface potential that activated the Raman scattering process is also determined as being $115 \mathrm{~nm}$. Based on our previous TEM studies, ${ }^{12}$ the calculated value does not correlate with neither diameter modulation nor surface roughness. The activation of these phonon modes is, therefore, tentatively attributed to resonant Raman scattering via defect states, e.g., due to point defects or structural defects located in the vicinity to the NW surfaces. Based on the performed comparison between the GaP and the GaP/GaNP NWs, the formation of these defects is concluded to be affected by the growth conditions (specifically the V/III ratio) and is not directly related to the presence of nitrogen.

The authors are thankful to B. Sernelius for fruitful discussions. Financial support by the Vetenskapsrådet (Swedish Research Council), Grant No. 621-2010-3815, is greatly appreciated. The nanowire growth was supported by the U.S. National Science Foundation under Grant Nos. DMR-0907652 and DMR-1106369. S.S. was partially funded by a Royal Government of Thailand Scholarship.

${ }^{1}$ J. Wang, M. S. Gudiksen, X. Duan, Y. Cui, and C. M. Lieber, Science 293, 1455 (2001).

${ }^{2}$ X. Duan, Y. Huang, Y. Cui, J. Wang, and C. M. Lieber, Nature 409, 66 (2001).

${ }^{3}$ M. S. Gudiksen, L. J. Lauhon, J. Wang, D. C. Smith, and C. M. Lieber, Nature 415, 617 (2002).

${ }^{4}$ N. Tajik, Z. Peng, P. Kuyanov, and R. R. LaPierre, Nanotechnology 22, 225402 (2011).

${ }^{5}$ H. K. Nosaki, K. Tomioka, S. Hara, K. Hiruma, J. Motohisa, and T. Fukui, Appl. Phys. Express 2, 035004 (2009).

${ }^{6}$ B. Tian, T. J. Kempa, and C. M. Lieber, Chem. Soc. Rev. 38, 16 (2009).

${ }^{7}$ S. Kumar, M. Jones, S. S. Lo, and G. D. Scholes, Small 3, 1633 (2007).

${ }^{8}$ B. M. Kayes, H. A. Atwater, and N. S. Lewis, J. Appl. Phys. 97, 114302 (2005).

${ }^{9}$ Y. J. Kuang, S. Sukrittanon, H. Li, and C. W. Tu, Appl. Phys. Lett. 100, 053108 (2012).

${ }^{10}$ A. Dobrovolsky, S. Sukrittanon, Y. J. Kuang, C. W. Tu, W. M. Chen, and I. A. Buyanova, Small 10(21), 4403-4408 (2014).

${ }^{11}$ A. Dobrovolsky, J. E. Stehr, S. L. Chen, Y. J. Kuang, S. Sukrittanon, C. W. Tu, W. M. Chen, and I. A. Buyanova, Appl. Phys. Lett. 101, 163106 (2012).

${ }^{12}$ S. Filippov, S. Sukrittanon, Y. Kuang, C. Tu, P. O. A. Persson, W. M. Chen, and I. A. Buyanova, Nano Lett. 14(9), 5264-5269 (2014).

${ }^{13}$ H. Yonezu, Semicond. Sci. Technol. 17, 762 (2002).

${ }^{14}$ I. A. Buyanova, W. M. Chen, and C. W. Tu, Semicond. Sci. Technol. 17, 815 (2002).

${ }^{15}$ W. Shan, W. Walukiewicz, K. M. Yu, J. Wu, J. W. Ager III, E. E. Haller, H. P. Xin, and C. W. Tu, Appl. Phys. Lett. 76, 3251 (2000).

${ }^{16}$ I. A. Buyanova, G. Pozina, J. P. Bergman, W. M. Chen, H. P. Xin, and C. W. Tu, Appl. Phys. Lett. 81, 52 (2002).

${ }^{17}$ I. A. Buyanova, M. Izadifard, A. Kasic, H. Arwin, W. M. Chen, H. P. Xin, Y. G. Hong, and C. W. Tu, Phys. Rev. B 70, 085209 (2004).

${ }^{18}$ A. Luque, A. Martí, and C. Stanley, Nat. Photonics 6, 146 (2012).

${ }^{19}$ R. E. Algra, M. A. Verheijen, M. T. Borgstrom, L.-F. Feiner, G. Immink, W. J. P. van Enckevort, E. Vlieg, and E. P. A. M. Bakkers, Nature 456, 369 (2008).

${ }^{20}$ K. A. Dick, P. Caroff, J. Bolinsson, M. E. Messing, J. Johansson, K. Deppert, L. R. Wallenberg, and L. Samuelson, Semicond. Sci. Technol. 25, 024009 (2010).

${ }^{21}$ R. Gupta, Q. Xiong, G. D. Mahan, and P. C. Eklund, Nano Lett. 3, 1745 (2003).

${ }^{22}$ D. Spirkoska, G. Abstreiter, and A. Fontcuberta i Morral, Semicond. Sci. Technol. 24, 113001 (2009). 
${ }^{23}$ I. Zardo, S. Conesa-Boj, F. Peiro, J. R. Morante, J. Arbiol, E. Uccelli, G. Abstreiter, and A. Fontcuberta i Morral, Phys. Rev. B 80, 245324 (2009).

${ }^{24}$ M. Montazeri, M. Fickenscher, L. M. Smith, H. E. Jackson, J. YarrisonRice, J. H. Kang, Q. Gao, H. H. Tan, C. Jagadish, Y. Guo, J. Zou, M.-E. Pistol, and C. E. Pryo, Nano Lett. 10, 880 (2010).

${ }^{25}$ N. G. Hormann, I. Zardo, S. Hertenberger, S. Funk, S. Bolte, M. Doblinger, G. Koblmuller, and G. Abstreiter, Phys. Rev. B 84, 155301 (2011).

${ }^{26}$ B. Ketterer, M. Heiss, E. Uccelli, J. Arbiol, and A. Fontcuberta i Morral, ACS Nano 5, 7585 (2011).

${ }^{27}$ E. G. Gadret, M. M. de Lima, Jr., J. R. Madureira, T. Chiaramonte, M. A. Cotta, F. Iikawa, and A. Cantarero, Appl. Phys. Lett. 102, 122101 (2013).

${ }^{28}$ I. A. Buyanova, W. M. Chen, E. M. Goldys, H. P. Xin, and C. W. Tu, Appl. Phys. Lett. 78, 3959 (2001).

${ }^{29}$ S. Yoon, M. J. Seong, J. F. Geisz, A. Duda, and A. Mascarenhas, Phys. Rev. B 67, 235209 (2003).

${ }^{30}$ V. Vorliček, I. Gregova, V. Riede, and H. Neumann, J. Phys. Chem. Solids 49, 797 (1988).

${ }^{31}$ P. Caroff, K. A. Dick, J. Johansson, M. E. Messing, K. Deppert, and L. Samuelson, Nat. Nanotechnol. 4, 50 (2008).
${ }^{32}$ R. E. Algra, M. Hocevar, M. A. Verheijen, I. Zardo, G. G. W. Immink, W. J. P. van Enckevort, G. Abstreiter, L. P. Kouwenhoven, E. Vlieg, and E. P. A. M. Bakkers, Nano Lett. 11, 1690 (2011).

${ }^{33}$ S. Assali, I. Zardo, S. Plissard, D. Kriegner, M. A. Verheijen, G. Bauer, A. Meijerink, A. Belabbes, F. Bechstedt, J. E. M. Haverkort, and E. P. A. M. Bakkers, Nano Lett. 13, 1559 (2013).

${ }^{34} \mathrm{Ch}$. Eckl, P. Pavone, J. Fritsch, and U. Schröder, in The Physics of Semiconductors, edited by M. Scheffler and R. Zimmermann (World Scientific, Singapore, 1996), Vol. 1, p. 229.

${ }^{35}$ H. E. Ruda and A. Shik, J. Appl. Phys. 100, 024314 (2006).

${ }^{36}$ A. Xiong, G. Chen, H. R. Gutierrez, and P. C. Eklund, Appl. Phys. A 85, 299 (2006).

${ }^{37}$ J. Johansson, L. S. Karlsson, C. P. T. Svensson, T. Mårtensson, B. A. Wacaser, K. Deppert, L. Samuelson, and W. Seifert, Nat. Mater. 5, 574 (2006).

${ }^{38}$ B. E. Sernelius, Surface Modes in Physics (Wiley-VCH, NY, 2001).

${ }^{39}$ S. Sahoo, M. S. Hu, C. W. Hsu, C. T. Wu, K. H. Chen, L. C. Chen, A. K. Arora, and S. Dhara, Appl. Phys. Lett. 93, 233116 (2008).

${ }^{40}$ S. E. Wu, S. Dhara, T. H. Hsueh, Y. F. Lai, C. Y. Wang, and C. P. Liu, J. Raman Spectrosc. 40, 2044 (2009). 